\title{
The association between physical activity and mental health during the first year of the COVID-19 pandemic: a systematic review
}

\author{
Priscila Marconcin ${ }^{1,2^{*}}$, André O. Werneck ${ }^{3}$, Miguel Peralta ${ }^{2,4}$, Andreas Ihle ${ }^{5,6,7}$, Élvio R. Gouveia ${ }^{8,9}$, Gerson Ferrari $^{10}$,
} Hugo Sarmento ${ }^{11}$ and Adilson Marques ${ }^{2,3}$

\begin{abstract}
Introduction: The Coronavirus disease-19 (COVID-19) pandemic affected countries worldwide and has changed peoples'lives. A reduction in physical activity and increased mental health problems were observed, mainly in the first year of the COVID-19 pandemic. Thus, this systematic review aims to examine the association between physical activity and mental health during the first year of the COVID-19 pandemic.

Methods: In July 2021, a search was applied to PubMed, Scopus, and Web of Science. Eligibility criteria included cross-sectional, prospective, and longitudinal study designs and studies published in English; outcomes included physical activity and mental health (e.g., depressive symptoms, anxiety, positive and negative effects, well-being).

Results: Thirty-one studies were included in this review. Overall, the studies suggested that higher physical activity is associated with higher well-being, quality of life as well as lower depressive symptoms, anxiety, and stress, independently of age. There was no consensus for the optimal physical activity level for mitigating negative mental symptoms, neither for the frequency nor for the type of physical activity. Women were more vulnerable to mental health changes and men were more susceptive to physical activity changes.

Conclusion: Physical activity has been a good and effective choice to mitigate the negative effects of the COVID-19 pandemic on mental health during the first year of the COVID-19 pandemic. Public health policies should alert for possibilities to increase physical activity during the stay-at-home order in many countries worldwide.
\end{abstract}

Keywords: Pandemic, SARS CoV-2, Exercise, Mental health, Anxiety

\section{Background}

The severe acute respiratory syndrome coronavirus-2 (SARS-CoV-2) is a highly contagious virus that infects humans and causes coronavirus disease-19 (COVID19), which is currently having a damaging impact on almost all countries worldwide [1]. To bring this pandemic to an end, a large share of the world needs to

${ }^{*}$ Correspondence: priscilamarconcin@fmh.ulisboa.pt

${ }^{2}$ CIPER, Faculdade de Motricidade Humana, Universidade de Lisboa,

Lisbon, Portugal

Full list of author information is available at the end of the article be immune to the virus, and the safest way to achieve this is with a vaccine. Fortunately, in December 2020 the vaccination started in the United Kingdom [2] and is currently pursued in different countries [3]. Until November 2021, 53.3\% of the world population has received at least one dose of a COVID-19 vaccine [4]. However, the number of infected people and deaths continues to grow [5]. The World Health Organization (WHO) published a weekly report and on 16th of November 2021 it was observed a increasing trend in new global weekly cases [6]. From the beginning of the pandemic, as a community mitigation strategy used 
to reduce the spread of COVID-19, most countries adopted the stay-at-home order as well as the stimulation of facemask wearing and hygiene habits $[7,8]$.

As a consequence of the stay-at-home strategies, mainly during the first year of the COVID-19 pandemic, studies had reported multiple behaviour changes. Some common impacts include disturbed eating behaviours [9], changes in alcohol consumption [10], and substance use [11]. A wide range of psychological outcomes has been observed during the virus outbreak, including a reduction in well-being as well as increases in depressive and anxiety symptoms $[12,13]$. Considering the need for social distancing measures, the investigation of possible factors that can mitigate the negative effects of social distancing on mental health should help the promotion of intervention strategies.

Physical activity (PA) is well recognised as a key factor for the prevention and management of mental illness, including mental disorders such as depression and anxiety as well as the promotion of mental health such as well-being [14, 15]. Nevertheless, globally, approximately $23 \%$ of adults and $81 \%$ of adolescents do not meet the WHO recommendations regarding PA for maintaining health [16-18]. This situation even worsened with the COVID-19 pandemic. Studies have demonstrated that PA declined and sedentary behaviour increased during the COVID-19 pandemic stay at home order in many countries, regardless of the subpopulation [19]. Different studies sought to investigate whether these changes in PA were associated with mental health indicators during the COVID-19 pandemic and a previous systematic review synthetised that PA is an effective strategy to face the psychological effects of the COVID-19 pandemic [20]. However, the previous review included articles published between 1 January 2019 , and 15 July 2020, before the second wave of the COVID-19 pandemic. Therefore, our systematic review aimed to update those findings and clarify if PA is associated with mental health during the first full year of the COVID-19 pandemic and to analyse if PA mitigates the effects of the stay-at-home order on mental health. We aimed to explore the first year of the COVID-19 pandemic because it was the period when restrictive orders were strictest when people were strongly encouraged to comply with the stay-at-home order.

\section{Methods \\ Design}

This systematic review focuses on peer-reviewed journal articles on the relationship of PA to mental health during the COVID-19 pandemic published until 30 July 2021.

\section{Data sources and searches}

A systematic review protocol was registered with the PROSPERO database on the 29th of January 2021 (IDCRD42021233921). A broad search strategy was employed. Searches were conducted on the 30th of January 2021, in the following electronic databases: PubMed, Scopus, and Web of Science. The search was performed in the three databases using the terms: (physical activity OR physical inactivity OR exercise OR training OR sport* OR fitness OR physical function* OR movement behavio* OR sedentary behavio*) AND (mental health OR psychological health OR depress" OR anxiety OR psychological function* OR mental function* OR wellbeing OR well-being OR burnout OR burn-out OR fear OR fears OR worries OR worry) AND (coronavirus disease OR COVID-19 OR SARS-CoV-2 OR lockdown OR shutdown OR quarantine OR confinement OR social isolation). These terms were searched in title and abstract of scientific articles. Additionally, cross-referencing search was performed in the full-text read of potentially included articles.

\section{Study selection}

Observational studies (cross-sectional, prospective, or longitudinal) were eligible for this review. Furthermore, studies were also required to meet the following criteria: (1) assessing PA by a validated instrument, (2) assessing mental health by a validated instrument, (3) presenting an analysis on the association between PA and mental health. Studies with samples including pregnant women, chronic disease patients, athletes, COVID-19 survivors, and frail older adults were excluded. Besides, studies reporting PA as a moderate or mediated variable were also excluded. Two co-authors screened titles and abstracts to identify articles that met the inclusion criteria. Two co-authors read the articles and decided whether they should be included in the analysis or excluded. The inclusion decision was consensual and in cases of disagreement, the decision was made by mutual agreement.

\section{Data extraction and synthesis of results}

Data extraction was completed independently by one coauthor. Data extracted from all studies included study details (author, year of publication, study design, recruitment processes, and date and location of the study); participant characteristics (sex, mean age); outcome and instruments, and main findings. A table was made for articles that analysed the association between PA and mental health among adults, and another table for the analyses of the association between PA and mental health among children and adolescents. 


\section{Quality assessment}

The risk of bias was assessed by two independent reviewers, using the Newcastle-Ottawa Scale (NOS) [21] which was also adapted for cross-sectional studies [22]. Therefore, we used the original scale for cohort studies and the adapted scale for the cross-sectional studies. The original scale varies between 0 and 9, while the adapted scale for cross-sectional studies varies between 0 and 10, with higher scores indicating research of better quality.

\section{Narrative synthesis}

Considering the heterogeneity of methods used for the estimation of the exposures and outcomes, it was not possible to conduct a meta-analysis. Therefore, we compared the findings across the included articles according to each outcome.

\section{Results}

\section{Results of the search}

From the database search, 734 records were identified. After removing duplicates, the titles and abstracts of 328 articles were screened concerning the eligibility criteria, and 205 were excluded. The full texts of the remaining 1237 articles were evaluated and 92 were excluded for the following reasons: sample characteristics $(n=24)$, data were not analysed regarding the association between PA and mental health variables $(n=23)$, review studies $(n=4)$, no valid instruments to assess PA $(n=31)$ and mental health $(n=6)$, the study was not in the period of the COVID-19 pandemic $(n=4)$. Thirty-one studies were included in this review, 27 about adults and old adults and 4 about children and adolescents. The flow diagram of study search and selection was created according to the Preferred Reporting Items for Systematic Reviews and MetaAnalyses (PRISMA) [23] and is presented in Fig. 1. The mean score of quality was $5.7 \pm 1.5$. More details are presented in Tables 1 and 2.

\section{The association between physical activity and mental health among adults and old adults}

The details of the association between PA and mental health among adults and old adults are summarised in Table 1, 27 studies were included [24-49, 51].

\section{Participants characteristics and date of filling the questionnaires}

The number of participants in the 27 included studies varied between 66 [50] and 14,715 [40] participants. Regarding sex, with exception of four studies [24, 31, $40,48]$ the majority included more women than men.
Concerning age, most articles presented the mean age range between 20 and 30 years [24, 25, 36, 37, 44, 48$50,56,57]$, two article presented mean age between 30 and 40 years [33, 43]; five articles presented mean age between 40 and 50 years [28, 32, 35, 41, 42]; and seven articles presented mean age above 50 years $[26,27,34$, $45-47,51]$. Four articles did not present mean age [31, $38-40]$, for one article the age ranged between 21 and 35 years [31], for another, the age ranged between 21 and 40 years [38], for another, the age ranged between 17 and 69 years [40], and in one the age ranged between 27 and 53 years [39]. The majority of studies reported, the sample filled out online questionnaires. One study used interviewed by telephone call [34]. Twenty four studies conducted a cross-sectional analysis and four a longitudinal analysis.

\section{Study location}

The studies were carried out on five different continents. Ten studies from Europe [24-28, 38, 39, 43, 44, 49], foure studies from Asia [36, 40, 46, 48, 50], seven studies from America [33-35, 37, 41, 51, 57] two studies from Africa $[31,56]$, two study from Oceania $[42,45]$, and two multicentre study [32, 47].

\section{Outcomes and instruments}

Concerning outcomes and instruments, 12 articles used the International Physical Activity Questionnaire (IPAQ) to assess PA and one article calculated an estimate of cardiorespiratory fitness (algorithm includes age, body composition, resting heart rate and PA) [57]. The others articles assessed PA with different instruments. Mental health included analyses of subjective well-being, sleep quality, depressive symptoms, anxiety, quality of life, psychological distress, motivation, resilience, affects (positive and negative), and health-related quality of life.

\section{Main findings}

Overall, all articles found a positive association between PA and better outcomes of mental health (e. g., depression, anxiety, well-being). Physical activity was explanatory variable for mental health [25]. Physical activity was positive associated with mental health [42, 49]. Articles that observed a decrease in PA during the stay-athome order also observed a decrease in well-being [47, 56], negative changes in depressive symptoms [57], and negative changes in anxiety and stress symptoms [45]. This relationship seems to be bidirectional, since participants who decrease in mental health had greater reduction in physical activity [37]. Inactive people had worse well-being, highest depression and enxiety compared with moderately active and very active participants [24, 


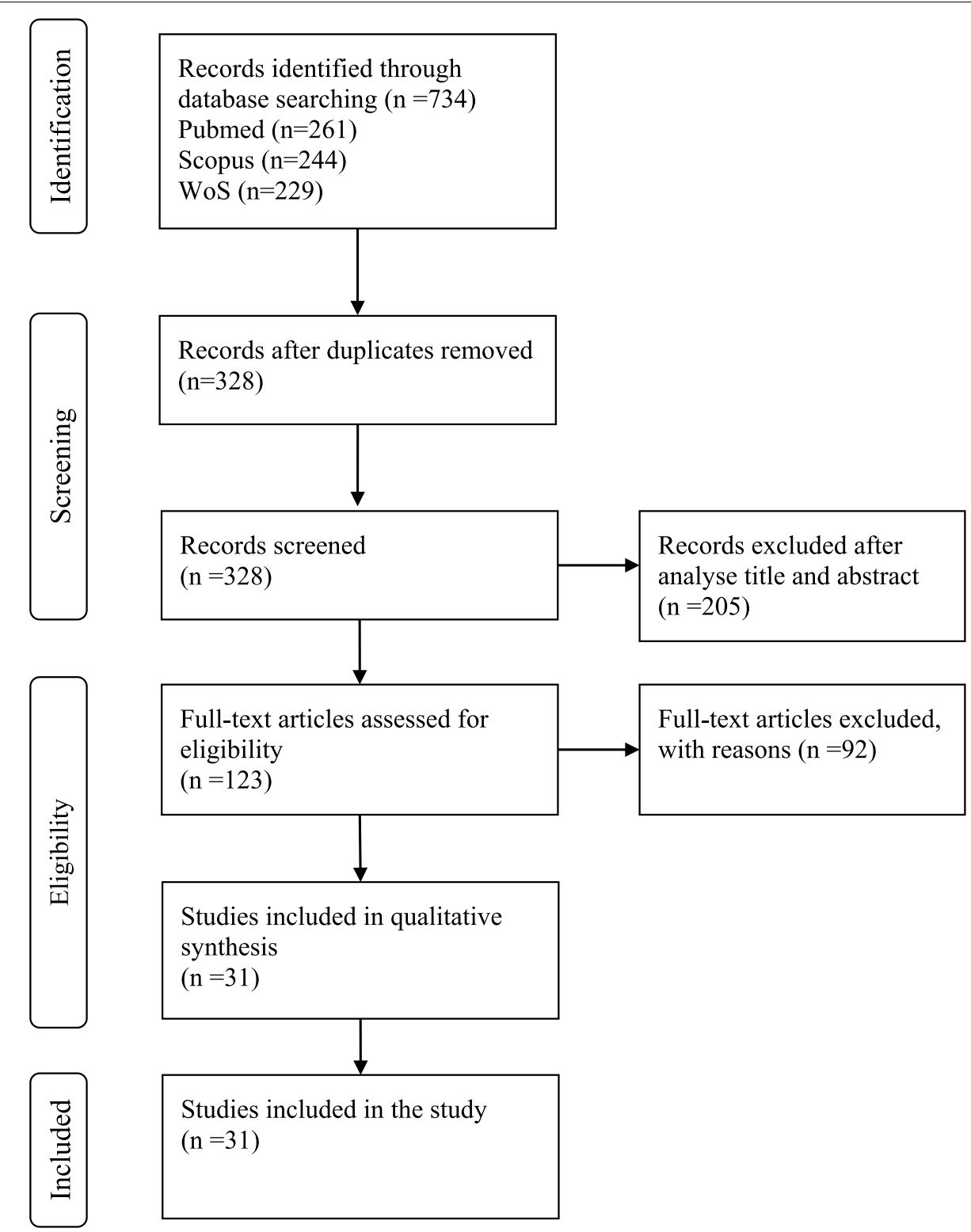

Fig. 1 Flow diagram of study selection

51]. Also, inactive old adults had more depressive symptoms [26]. On the other hand, participants sufficiently active reported significantly lower depression and anxiety and higher life satisfaction. Moreover, it was found that exercise intensity seems to be important. Two studies founded that depression was significantly negatively correlated with moderate-intensity PA but not vigorous and walking/light exeeercuse [36, 40]. Another one found that vigorous PA better predicted depressive symptoms than moderate PA [27]. Three studies suggested that the threshold of PA should be done to fells the benefits on mental health $[28,39,50]$. At least $4 \mathrm{~h}$ of MVPA reduced by $49 \%$ odds of depressive symptoms [28], and at leas 477 METs-min/week was associated with a $33 \%$ decrease in the probability of depressive symptoms [39]. On the other hand, a non-significant association was found between PA and anxiety [35], and between PA and health-related quality of life [46]. One study found that the decrease in mental wellbeing and increase in perceived stress was not related to changes in PA [44]. 


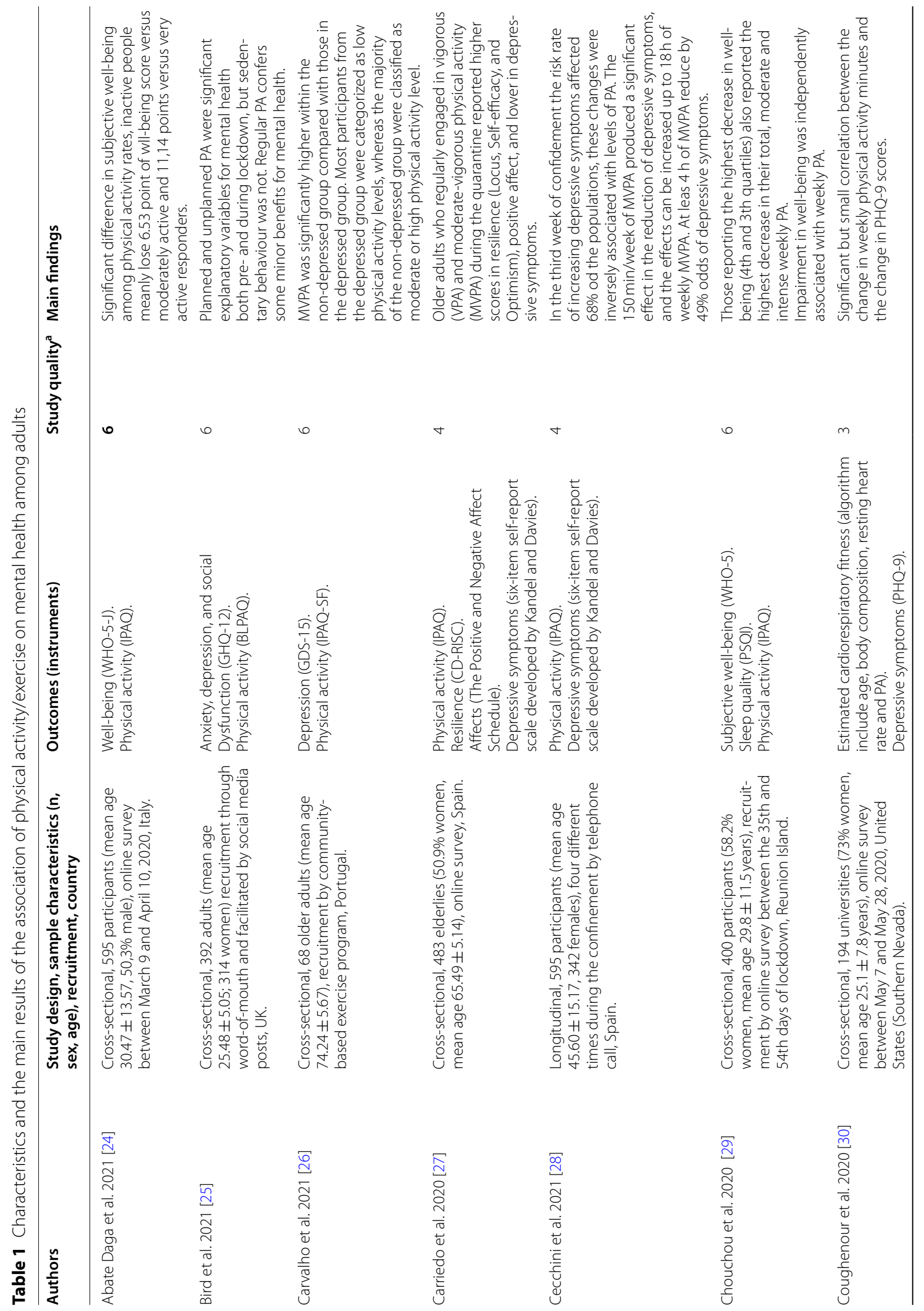




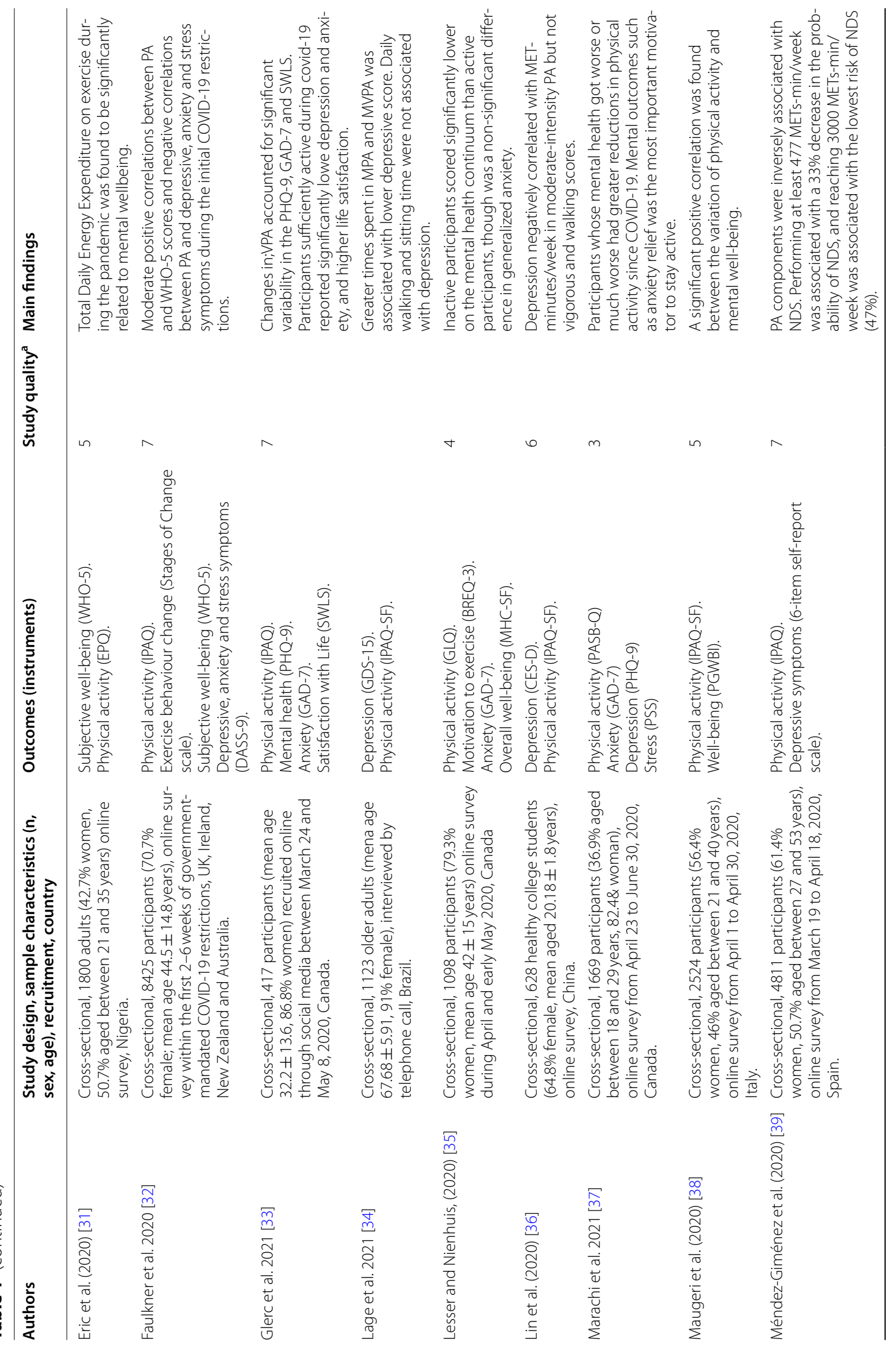




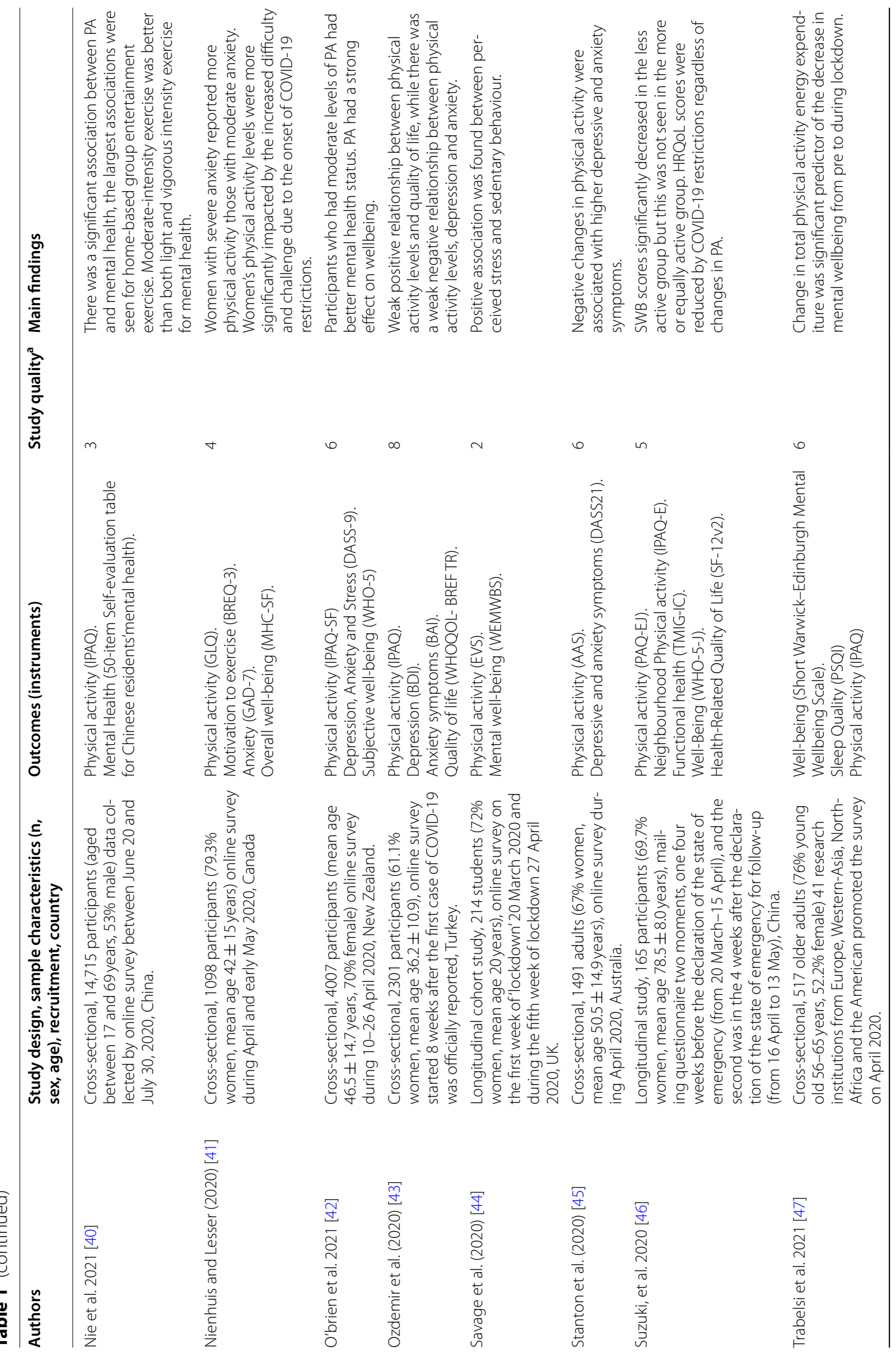




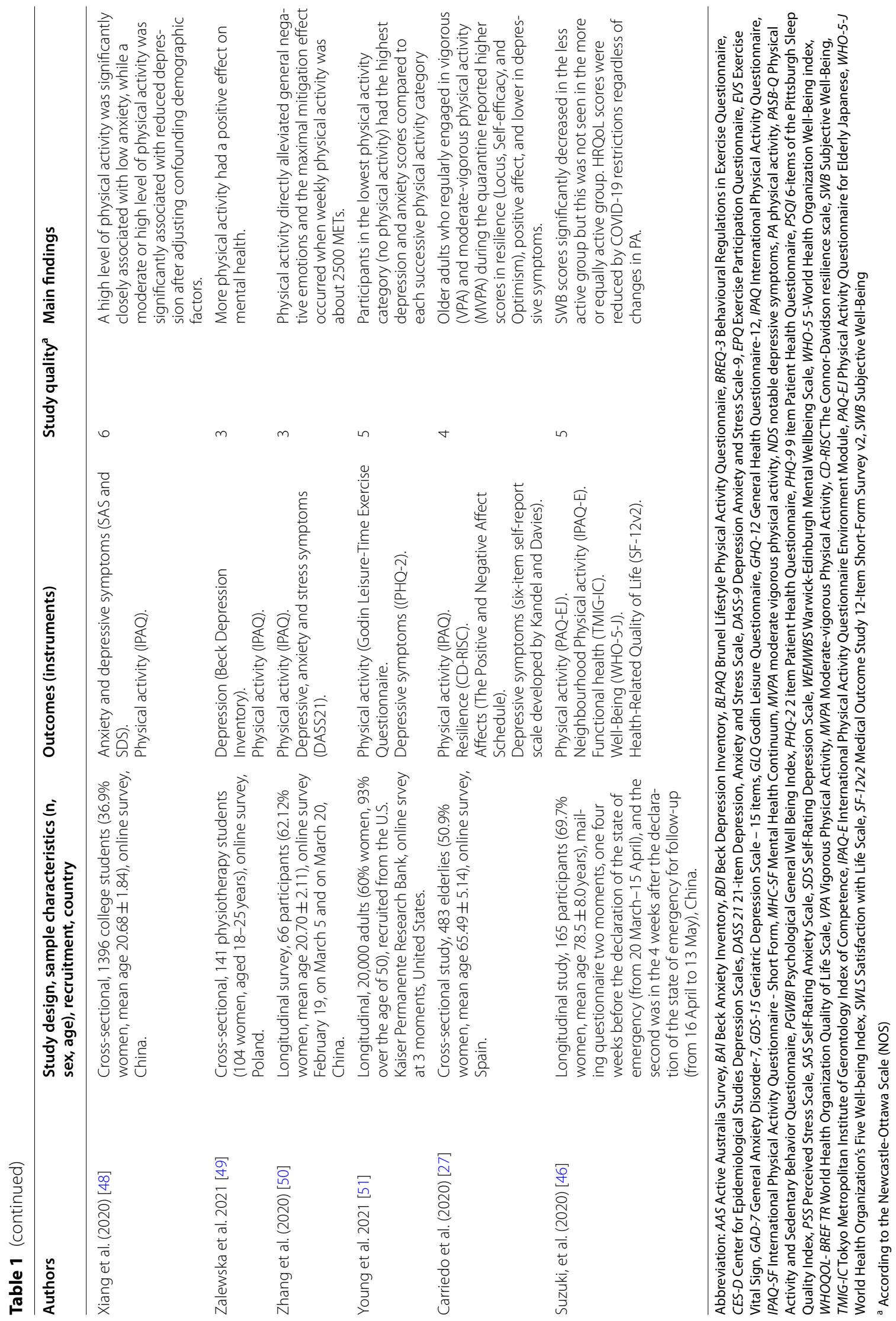




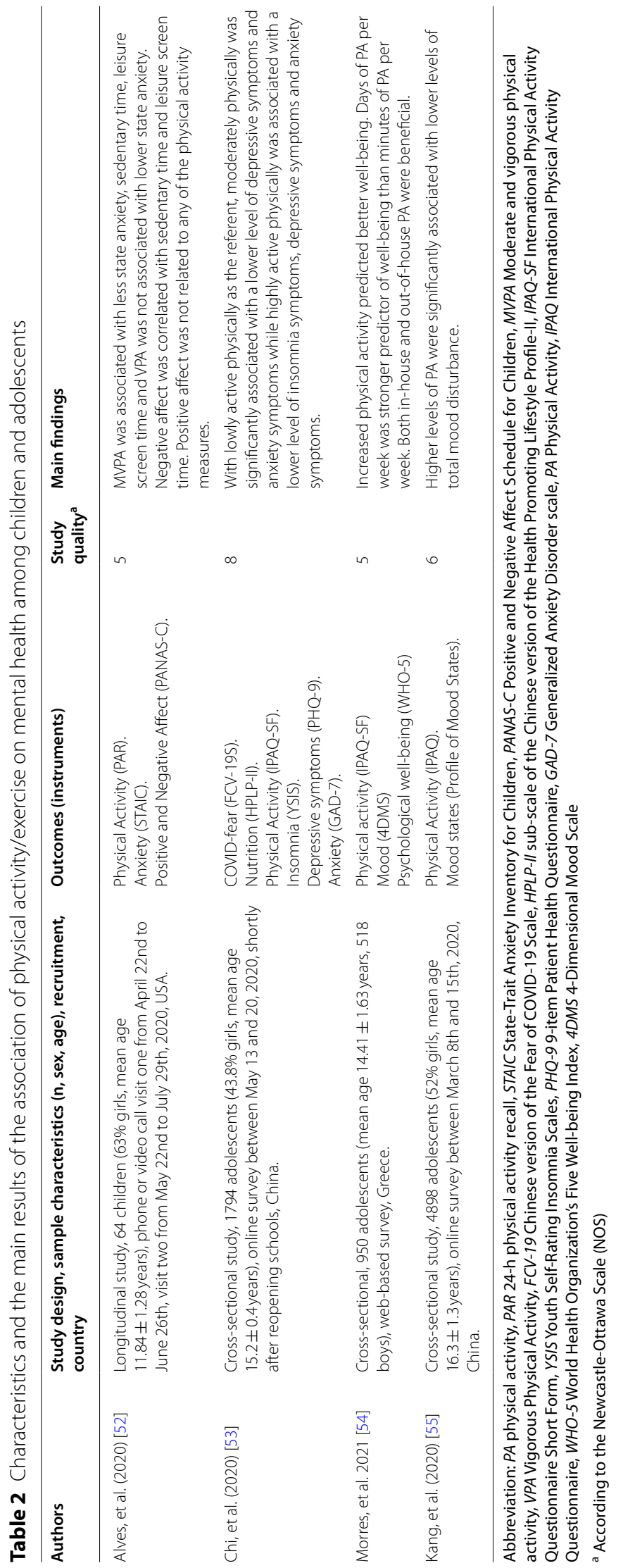




\section{The association between physical activity and mental} health among children and adolescents

The details of the association between PA and mental health among children and adolescents are shown in Table 2, four studies were included [52-55].

\section{Participants characteristics and date of filling the questionnaires}

The number of participants ranged between 64 and 4898 children and adolescents. More girls than boys participated in the studies. The mean ages were 11, 14, 15, and 16 years old. One study was longitudinal and presents two moments of assessment, also opting for phone or video calls to collect the data [52]. The other three studies were cross-sectional, and collected data by online surveys.

\section{Study location}

One study was from the USA [52], one from Greece [54] and the other two were from China $[53,55]$.

\section{Outcomes and instruments}

Three studies assessed PA by the International physical activity questionnaire (IPAQ) questionnaire [53-55], and another one used a 24-h physical activity recall [52]. Regarding mental health, different outcomes were assessed, such as anxiety, positive and negative affect, insomnia, depressive symptoms, psychological wellbeing and mood states.

\section{Main findings}

Moderate physical activity was associated with less state anxiety $[52,53]$. Positive affect was not related to physical activity [52]. Higher levels of physical activity were also significantly associated with lower levels of total mood disturbance [55]. Regarding the dose of physical activity, days of physical activity per week was stronger predictor of well-being than minutes of physical activity per week [54].

\section{Discussion}

This systematic review focuses on the association between PA and mental health during the first year of the COVID-19 pandemic. In particular, we sought to answer if PA mitigates the effects of the stay-at-home order on mental health. The COVID-19 pandemic generated numerous challenges for public health, particularly the significant burden of mental health in the population $[58,59]$. In addition, PA has been recognized as an effective mitigation strategy for improving mental health [60]. The COVID-19 pandemic has been affecting all continents in the world, at different scales. This study analysed
31 research articles, 27 about adults and old adults and 4 about children and adolescents. The articles are mainly based on cross-sectional studies and five are longitudinal studies. In nearly all of the studies comprised in the present systematic review, investigators used online surveys as the main procedure to collect data. Overall, the studies suggested that higher PA is associated with less negative mental health symptoms, such as depression, anxiety, well-being, and fear, independently of age.

The studies observed that women showed more depressive symptoms than men [28, 39, 50, 53, 55], and this trend increases with age [24] Furthermore, individuals with a lower level of masculinity traits (not specifically females) increased risk of developing depression [36], and women experienced more generalised anxiety [41]. The reduction of PA levels may mostly influence the mental well-being of females [38, 49]. Those findings are expected since the literature is consistent in signalising sex differences in most mental disorders [61]. On the whole, the prevalence rates of anxiety and depression were both higher than the rates found in previous studies before the COVID-19 pandemic [36, 37, 43, 45, 48, 57], which highlights a worsening in mental health during the first year of the COVID-19 pandemic. Regarding age, younger individuals experienced significantly higher anxiety and depression, also income influenced mental health, lower-income participants present worse mental health [37].

Five articles conducted a longitudinal study. Among those studies, four enrolled adults and old adults [28, $44,46,50,51]$ and in one the sample comprised children [52]. Among the studies that have collected measures before and after the stay-at-home order, both observed a significant reduction in PA [44, 46]. Two study collected measures after the stay-at-home order and during this period physical activity mean score decreased minimally [51], and depressive symptoms increase as the weeks of isolation go [28]. Other studies also reported a reduction in self-reported PA [26, 33, 34, 37, 38, 42, 43, 45, 56, 57] and increase in sedentary behaviour [25, 26, 34]. Individuals who reported larger decrease in MVPA pre to during COVID-19 reported relatively poorer mental health [33]. Participants whose mental health got "worse" or "much worse" had greater reductions in physical activity [37]. Also, increased levels of physical activity were associated with stronger effects on wellbeing [42]. The reduction was more pronounced in men than women [32, 38, 44], in vigorous PA [38], and between those with lower health-related quality of life scores before the COVID-19 pandemic [46]. The possible explanation for this sex-difference is that men are more engaged in group/community PA and sports in clubs or gyms, and those were more impacted by the COVID-19 restrictions. Also, women 
are more engaged in low and moderate physical activities, which can be done at home. Besides, women spent more time in housework activities. Women without changes in childcare provision reported more opportunities to be physically active [41]. The same sex-differences were observed in an Italian study [62]. Increases in PA were observed for a minority, but the majority of the respective study samples demonstrating a positive change were individuals who did not meet recommended PA guidelines before the COVID-19 pandemic [32, 38]. Additional reasons could be an increase in awareness for health issues and more time to pursue PA during the stay-athome order [32]. These behaviour changes can help to maintain a more active lifestyle during the pandemic. Another study found an increase of $40 \%$ in PA in a sample that was already active before the COVID-19 pandemic [35]. PA could be used as a coping strategy to deal with the consequences of the pandemic. The place where individuals prefer to practice PA seems to be important, since active participants reported greater connectedness to nature and nature relatedness than the inactive population [35].

There was no consensus across studies for the optimal PA levels for mitigating negative mental symptoms. The more the physical activity is frequent and vigorous, the best people feel themselves [24]. Among Chinese students, 2500 METs minute/week of PA every week was the optimal dose to alleviate negative emotion [50]. On the other hand, a Spanish community sample study showed that 477 METs-minute/week was associated with a 33\% decrease in the probabilities of notable depressive symptoms [39]. The difference between the values must be relativised considering the samples' characteristics. The first one concerns students with a mean age of 20 years [50], and the second one concerns a community sample with a mean age of 43.2 years for women and 40.5 years for men [39]. In addition, it is claimed that at least 3000 METsminute/week reduce the odds of depressive symptoms by $47 \%$ [39]. These studies used the IPAQ to assess PA, and according to IPAQ, to reach a minimally active category at least 600 METs minute/week is needed [63]. The American College of Sports Medicine also recommends for healthy adults aged 18-65 years at least 600 METs minute/week but did not specify the minimum dose to prevent depressive symptoms $[14,64]$.

Studies also examined the association between PA and mental health according to PA intensity. Moderate-intensity PA (e.g., walking or jogging on a treadmill, using an elliptical trainer, cleaning house) is associated with better mental health outcomes than vigorous-intensity PA $[36,40]$, and light-intensity PA [40]. On the other hand, vigorous-intensity PA better predicted depressive symptoms than moderate-intensity PA; also the effect size was higher for the association between vigorous-intensity PA and level of resilience compared with moderate-intensity PA [27]. One study found that performing high PA levels has no positive effect on depressive symptoms [39]. Another study explored the type of PA and showed that stretching and resistance training were associated with lower anxiety, and three types of PA (household chores, stretching, and resistance training) were associated with lower depression symptoms [48].

One study explored the association of specific types of physical exercise and mental health, and founded that home-based group entertainment exercise, rope skipping and badminton, Chinese traditional sports, video dancing and sensory-motor games present a greater reduction in mental health than others types [40].

Sedentary behaviour was observed in few studies and contradictory findings were observed. No association between sedentary time and depressive symptoms was observed [26, 36]. However, other studies have shown that sedentary behaviour was associated with poorer mental health [25], well-being [32] and perceived stress [44].

Concerning the association between PA and mental health outcomes among children and adolescents, only four articles were selected. Some issues must be highlighted. This population had to face, beyond the reality that changed from the COVID-19 pandemic, the changes in the education system such as online learning became the main learning method for students and uncertainty of academic development, which probably caused more anxiety level $[52,53]$. Both moderate and highly active groups were significantly associated with less depressive symptoms [53] and anxiety [52, 53], and only the most active adolescents reported significantly lower insomnia symptoms [53] and better mood states [55]. Days of physical activity per week was stronger predictor of well-being than minutes of physical activity per week [54].

Regarding old-age samples, the studies mentioned the particular vulnerability to changes in social circumstances, and highlight the importance of health-related quality of life [46] and levels of resilience [27] to deal with the consequences of the COVID-19 pandemic on the PA level. The stay-at-home order can cause greater distress and feelings of sadness, considered specific risk factor for depressive symptoms [34]. Mental health and physical activity decrease pre to during stay-at-home order [47]. In a group that were previously regular participants of a formal exercise program, MVPA was significantly higher within the non-depressed group compared with depressed group [26]. Being active previously of COVID19 confinement did not prevent $30.4 \%$ of Brazilian older adults from having depressive symptoms, but these 
results is much lower than prevalence of depression in Brazilian general population, which is 68\% [34].

The present systematic review had some limitations that must be mentioned. First, the studies present correlative analyses, not causal ones, thus randomised controlled trials must be conducted in the context of the COVID-19 pandemic and the stay-at-home order to clarify the direction of the association. However, beyond the COVID-19 context, randomised controlled trials showed that PA interventions show beneficial effects on mental health outcomes such as depression and anxiety [65]. Thus, a nuanced perspective particularly during the COVID-19 context in future research is needed. Moreover, the included studies with community samples were limited, and the analyses were mostly based on convenience samples with college students, which had specific characteristics and low mean age. Thus, future research needs to focus on representative study samples.

\section{Conclusion}

This review helps to clarify the positive association between PA and mental health during the first year of the COVID-19 pandemic, especially considering the effects of the stay-at-home order worldwide. Although there is an association between increased PA and improved mental health, further studies are needed, specifically randomised clinical trials, to identify the direction of this relationship, and what kind of PA, intensity, and frequency are most indicated to maximise the effects. Also, an investigation to examine the association during the second year of the COVID-19 pandemic is needed. The impact of the COVID-19 pandemic on mental health may be continuous and long-term [66, 67]. Thus, public health agencies must provide timely and effective interventions, in which PA and exercise should be a priority.

\section{Abbreviations \\ COVID-19: Coronavirus disease-19; SARS-CoV-2: Severe acute respiratory syn- drome coronavirus-2; PA: Physical activity; WHO: World Health Organization;} IPAQ: International physical activity questionnaire; MET: Metabolic equivalent.

\section{Acknowledgements}

Al acknowledges support from the Swiss National Centre of Competence in Research LIVES - Overcoming vulnerability: life course perspectives, granted by the Swiss National Science Foundation (grant number: 51NF40-185901). Al acknowledges support from the Swiss National Science Foundation (grant number: 10001C_189407). André Werneck is supported by the São Paulo Research Foundation (FAPESP) with a PhD scholarship (FAPESP process: 2019/24124-7). This paper presents independent research. The views expressed in this publication are those of the authors and not necessarily those of the acknowledged institutions.

\section{Authors' contributions}

All authors were involved in drafting the article or revising it critically for important intellectual content, and all authors approved the final version to be submitted for publication. PM, MP and AM had full access to all of the data in the study and take responsibility for the integrity of the data and the accuracy of the data analysis. PM, MP and AM, study conception and design. PM and MP acquisition of data. PM, MP and AM, analysis and interpretation of data.

\section{Funding}

No funding.

\section{Availability of data and materials}

Data sharing is not applicable to this article as no datasets were generated or analysed during the current study.

\section{Declarations}

Ethics approval and consent to participate

Not applicable.

\section{Consent for publication}

Not applicable.

\section{Competing interests}

The authors declare that they have no competing interests.

\section{Author details}

${ }^{1}$ KinesioLab, Research Unit in Human Movement Analysis, Insituto Piaget, Almada, Portugal. ${ }^{2} \mathrm{CIPER}$, Faculdade de Motricidade Humana, Universidade de Lisboa, Lisbon, Portugal. ${ }^{3}$ Center for Epidemiological Research in Nutrition and Health, Department of Nutrition, School of Public Health, São Paulo, Brazil. ${ }^{4}$ ISAMB, Universidade de Lisboa, Lisbon, Portugal. ${ }^{5}$ Center for the Interdisciplinary Study of Gerontology and Vulnerability, University of Geneva, Geneva, Switzerland. ${ }^{6}$ Swiss National Centre of Competence in Research LIVES - Overcoming vulnerability: Life course perspectives, Lausanne and Geneva, Switzerland. ${ }^{7}$ Department of Psychology, University of Geneva, Geneva, Switzerland. ${ }^{8}$ Universidade da Madeira, Funchal, Portugal. ${ }^{9}$ Interactive Technologies Institute, LARSyS, Funchal, Portugal. ${ }^{10}$ Laboratorio de Rendimiento Humano, Grupo de Estudio en Educación, Actividad Física y Salud (GEEAFyS), Universidad Católica del Maule, Talca, Chile. ${ }^{11}$ University of Coimbra, Research Unit for Sport and Physical Activity (CIDAF). Faculty of Sport Sciences and Physical Education, Coimbra, Portugal.

Received: 28 October 2021 Accepted: 17 January 2022

Published online: 01 February 2022

\section{References}

1. Conti P, Caraffa A, Gallenga CE, Kritas SK, Frydas I, Younes A, et al. The British variant of the new coronavirus-19 (Sars-Cov-2) should not create a vaccine problem. J Biol Regul Homeost Agents. 2020;35(1).

2. Ritchie H, Ortiz-Ospina E.; Beltekian D., Mathieu E., Hasell J., Macdonald B., Giattino C. and Roser M. Coronavirus (COVID-19) Vaccinations 2021 Available from: https://ourworldindata.org/covid-vaccinations.

3. Organization WH. Coronavirus disease (COVID-19): Vaccines 2021 [Available from: https://www.who.int/news-room/q-a-detail/coronavirus-disea se-(covid-19)-vaccines?adgroupsurvey $=\{$ adgroupsurvey $\}$ gclid $=$ CjwKC AiA1eKBBhBZEiwAX3gglwcwqCmOIN-6welx1Msuxpy-YUyvsWAV-BYZOJ 1g1qS0oUYbLEPiZxoC9UQQAvD_BwE.

4. Our World in Data. Coronavirus (COVID-19) Vaccinations. 2021.

5. Organization WH. WHO Coronavirus Disease (COVID-19) Dashboard 2021 [Available from: https://covid19.who.int/?gclid=CjwKCAiAmrOBBhA OEiwArn3mfDVdryFCBj-_IYP99HuMas-LYQzoVDYdfWMBkTmfFA8QVtmS OncCTRoCiFIQAvD_BwE.

6. WHO. Weekly epidemiological update on COVID-19 16 November 2021. 2021.

7. European Centre for Disease Prevention and Control. Data on country response measures to COVID-19 2021 [Available from: https://www. ecdc.europa.eu/en/publications-data/download-data-response-measu res-covid-19.

8. Moreland A, Herlihy C, Tynan MA, Sunshine G, McCord RF, Hilton C, et al. Timing of state and territorial COVID-19 stay-at-home orders and changes in population movement-United States, march 1-may 31, 2020. Morb Mortal Wkly Rep. 2020;69(35):1198. 
9. Valentin F, Sylvain I, Oulmann Z, Rachel FR, Joël B, Pierre-Michel L, et al. COVID-19 pandemic lockdown and problematic eating behaviors in a student population. J Behav Addict. 2020;9(3):826-35.

10. Chodkiewicz J, Talarowska M, Miniszewska J, Nawrocka N, Bilinski P. Alcohol consumption reported during the COVID-19 pandemic: the initial stage. Int J Environ Res Public Health. 2020;17(13):4677.

11. Wei Y, Shah R. Substance use disorder in the COVID-19 pandemic: a systematic review of vulnerabilities and complications. Pharmaceuticals. 2020;13(7):155

12. Salari N, Hosseinian-Far A, Jalali R, Vaisi-Raygani A, Rasoulpoor S, Mohammadi $M$, et al. Prevalence of stress, anxiety, depression among the general population during the COVID-19 pandemic: a systematic review and meta-analysis. Glob Health. 2020;16(1):57.

13. Chen $Q$, Li M, Wang Y, Zhang L, Tan X. Changes in anxiety, depression, and stress in one week and one month later after the Wuhan shutdown against the COVID-19 epidemic. Disast Med Public Health Prepared. 2021;1-8.

14. Teychenne M, White RL, Richards J, Schuch FB, Rosenbaum S, Bennie JA. Do we need physical activity guidelines for mental health: what does the evidence tell us? Ment Health Phys Act. 2020;18:100315.

15. Marques A, Peralta M, Martins J, Catunda R, Matos MG, Saboga NL. Associations between physical activity and self-rated wellbeing in European adults: a population-based, cross-sectional study. Prev Med. 2016;91:18-23.

16. WHO. Physical Inactivity: a global public health problem 2021 [Available from: https://www.who.int/ncds/prevention/physical-activity/inactivityglobal-health-problem/en/

17. Marques A, Henriques-Neto D, Peralta M, Martins J, Demetriou Y, Schönbach DMI, et al. Prevalence of physical activity among adolescents from 105 low, middle, and high-income countries. Int J Environ Res Public Health. 2020;17(9).

18. Guthold R, Stevens GA, Riley LM, Bull FC. Worldwide trends in insufficient physical activity from 2001 to 2016: a pooled analysis of 358 population-based surveys with 1.9 million participants. Lancet Glob Health. 2018;6(10):e1077-e86.

19. Stockwell S, Trott M, Tully M, Shin J, Barnett Y, Butler L, et al. Changes in physical activity and sedentary behaviours from before to during the COVID-19 pandemic lockdown: a systematic review. BMJ Open Sport Exerc Med. 2021;7(1):e000960.

20. Violant-Holz V, Gallego-Jiménez MG, González-González CS, MuñozViolant S, Rodríguez MJ, Sansano-Nadal O, et al. Psychological health and physical activity levels during the COVID-19 pandemic: a systematic review. Int J Environ Res Public Health. 2020:17(24):9419.

21. Wells GA, Shea B, O'Connell D, Peterson J, Welch V, Losos M, et al. The Newcastle-Ottawa Scale (NOS) for assessing the quality if nonrandomized studies in meta-analyses. Available from: http://www.ohri.ca/ programs/clinical_epidemiology/oxford.htm [cited 2021 Dec 28].

22. Modesti PA, Reboldi G, Cappuccio FP, Agyemang C, Remuzzi G, Rapi S, et al. Panethnic differences in blood pressure in Europe: a systematic review and Meta-analysis. PLoS One. 2016;11(1):e0147601.

23. Moher D, Liberati A, Tetzlaff J, Altman DG. Preferred reporting items for systematic reviews and meta-analyses: the PRISMA statement. BMJ. 2009;339:b2535.

24. Abate Daga F, Agostino S, Peretti S, Beratto L. The impact of physical activity rate on subjective well-being among North-Western Italian population during COVID-19 nationwide lockdown. J Sports Med Phys Fitness. 2021

25. Bird JM, Karageorghis $\mathrm{Cl}$, Hamer M. Relationships among behavioural regulations, physical activity, and mental health pre- and during COVID19 UK lockdown. Psychol Sport Exerc. 2021;55:101945.

26. Carvalho J, Borges-Machado F, Pizarro AN, Bohn L, Barros D. Home confinement in previously active older adults: a cross-sectional analysis of physical fitness and physical activity behavior and their relationship with depressive symptoms. Front Psychol. 2021;12:643832.

27. Carriedo A, Cecchini JA, Fernandez-Rio J, Méndez-Giménez A. COVID19, psychological well-being and physical activity levels in older adults during the Nationwide lockdown in Spain. Am J Geriatr Psychiatry. 2020;28(11):1146-55.

28. Cecchini JA, Carriedo A, Fernández-Río J, Méndez-Giménez A, González C, Sánchez-Martínez B, et al. A longitudinal study on depressive symptoms and physical activity during the Spanish lockdown. Int J Clin Health Psychol. 2021;21(1):100200.
29. Chouchou F, Augustini M, Caderby T, Caron N, Turpin NA, Dalleau G. The importance of sleep and physical activity on well-being during COVID-19 lockdown: Reunion island as a case study. Sleep Med. 2021;77:297-301.

30. Coughenour C, Gakh M, Pharr JR, Bungum T, Jalene S. Changes in depression and physical activity among college students on a diverse campus after a COVID-19 stay-at-home order. J Community Health. 2021;46(4):758-66.

31. Eric NO, Oghenebrorien AE, Onogimesike AT, Suoke OP. Mental wellbeing and exercise participation during covid-19 pandemic among adult residents of Nigeria's Niger delta. Int J Hum Move Sports Sci. 2020;8(6):534-42

32. Faulkner J, O'Brien WJ, McGrane B, Wadsworth D, Batten J, Askew CD, et al. Physical activity, mental health and well-being of adults during initial COVID-19 containment strategies: a multi-country cross-sectional analysis. J Sci Med Sport. 2020;24(4):320-6.

33. Gierc M, Riazi NA, Fagan MJ, Di Sebastiano KM, Kandola M, Priebe CS, et al Strange days: adult physical activity and mental health in the first two months of the COVID-19 pandemic. Front Public Health. 2021;9:567552.

34. Lage A, Carrapatoso S, de Queiroz S, Neto E, Gomes S, Soares-Miranda L, et al. Associations between depressive symptoms and physical activity intensity in an older adult population during COVID-19 lockdown. Front Psychol. 2021;12:644106.

35. Lesser IA, Nienhuis CP. The Impact of COVID-19 on Physical Activity Behavior and Well-Being of Canadians. Int J Environ Res Public Health. 2020:17(11):3899.

36. Lin J, Guo T, Becker B, Yu Q, Chen ST, Brendon S, et al. Depression is associated with moderate-intensity physical activity among college students during the COVID-19 pandemic: differs by activity level, gender and gender role. Psychol Res Behav Manag. 2020;13:1123-34.

37. Marashi MY, Nicholson E, Ogrodnik M, Fenesi B, Heisz JJ. A mental health paradox: mental health was both a motivator and barrier to physical activity during the COVID-19 pandemic. PLoS One. 2021;16(4):e0239244.

38. Maugeri G, Castrogiovanni P, Battaglia G, Pippi R, D’Agata V, Palma A, et al. The impact of physical activity on psychological health during Covid-19 pandemic in Italy. Heliyon. 2020;6(6):e04315.

39. Méndez-Giménez A, Cecchini JA, Fernández-Río J, Carriedo A. Physical activity and prevention of depressive symptoms in the Spanish population during confinement due to COVID-19. Psicothema. 2021;33(1):111-7.

40. Nie YJ, Ma YY, Wu YK, Li JH, Liu T, Zhang C, et al. Association between physical exercise and mental health during the COVID-19 outbreak in China: a Nationwide cross-sectional study. Front Psychiatry. 2021;12:722448.

41. Nienhuis CP, Lesser IA. The Impact of COVID-19 on Women's Physical Activity Behavior and Mental Well-Being. Int J Environ Res Public Health. 2020;17(23):9036.

42. O'Brien L, Forster J. Physical activity supporting connection to nature, and helping to maintain wellbeing during the Covid-19 restrictions in England. Int J Environ Res Public Health. 2021;18(9):4585.

43. Ozdemir F, Cansel N, Kizilay F, Guldogan E, Ucuz I, Sinanoglu B, et al. The role of physical activity on mental health and quality of life during COVID19 outbreak: a cross-sectional study. Eur J Integrat Med. 2020;40:101248.

44. Savage MJ, James R, Magistro D, Donaldson J, Healy LC, Nevill M, et al. Mental health and movement behaviour during the COVID-19 pandemic in UK university students: prospective cohort study. Ment Health Phys Act. 2020;19.

45. Stanton R, To QG, Khalesi S, Williams SL, Alley SJ, Thwaite TL, et al. Depression, Anxiety and Stress during COVID-19: Associations with Changes in Physical Activity, Sleep, Tobacco and Alcohol Use in Australian Adults. Int J Environ Res Public Health. 2020;17(11):4065.

46. Suzuki Y, Maeda N, Hirado D, Shirakawa T, Urabe Y. Physical Activity Changes and Its Risk Factors among Community-Dwelling Japanese Older Adults during the COVID-19 Epidemic: Associations with Subjective Well-Being and Health-Related Quality of Life. Int J Environ Res Public Health. 2020;17(18):6591.

47. Trabelsi K, Ammar A, Masmoudi L, Boukhris O, Chtourou H, Bouaziz $B$, et al. Sleep quality and physical activity as predictors of mental wellbeing variance in older adults during COVID-19 lockdown: ECLB COVID-19 international online survey. Int J Environ Res Public Health. 2021;18(8):4329.

48. Xiang MQ, Tan XM, Sun J, Yang HY, Zhao XP, Liu L, et al. Relationship of physical activity with anxiety and depression symptoms in Chinese college students during the COVID-19 outbreak. Front Psychol. 2020;11:582436. 
49. Zalewska A, Gałczyk M, Sobolewski M, Białokoz-Kalinowska I. Depression as compared to level of physical activity and internet addiction among polish physiotherapy students during the COVID-19 pandemic. Int J Environ Res Public Health. 2021;18(19):10072.

50. Zhang Y, Zhang H, Ma X, Di Q. Mental Health Problems during the COVID-19 Pandemics and the Mitigation Effects of Exercise: A Longitudinal Study of College Students in China. Int J Environ Res Public Health. 2020;17(10):3722.

51. Young DR, Hong BD, Lo T, Inzhakova G, Cohen DA, Sidell MA. The longitudinal associations of physical activity, time spent outdoors in nature and symptoms of depression and anxiety during COVID-19 quarantine and social distancing in the United States. Prev Med. 2021;154:106863.

52. Alves JM, Yunker AG, DeFendis A, Xiang AH, Page KA. Associations between affect, physical activity, and anxiety among US children during COVID-19. medRxiv. 2020.

53. Chi X, Liang K, Chen ST, Huang Q, Huang L, Yu Q, et al. Mental health problems among Chinese adolescents during the COVID-19: the importance of nutrition and physical activity. Int J Clin Health Psychol. 2020;21:100218.

54. Morres ID, Galanis E, Hatzigeorgiadis A, Androutsos O, Theodorakis Y. Physical activity, sedentariness, eating behaviour and well-being during a COVID-19 lockdown period in Greek adolescents. Nutrients. 2021;13(5):1449.

55. Kang S, Sun Y, Zhang X, Sun F, Wang B, Zhu W. Is physical activity associated with mental health among Chinese adolescents during isolation in COVID-19 pandemic? J Epidemiol Global Health. 2020;1 (1):26-33.

56. Chouchou F, Augustini M, Caderby T, Caron N, Turpin NA, Dalleau G. The importance of sleep and physical activity on well-being during COVID-19 lockdown: Reunion island as a case study. Sleep Med. 2020:297-301.

57. Coughenour C, Gakh M, Pharr JR, Bungum T, Jalene S. Changes in depression and physical activity among college students on a diverse campus after a COVID-19 stay-at-home order. J Community Health. 2020:758-66.

58. Lima CKT, Carvalho PMM, Lima I, Nunes J, Saraiva JS, de Souza RI, et al. The emotional impact of coronavirus 2019-nCoV (new coronavirus disease). Psychiatry Res. 2020;287:112915.

59. Hahad O, Gilan DA, Daiber A, Münzel T. Public mental health as one of the key factors in dealing with COVID-19. Gesundheitswesen. 2020;82(5):389-91.

60. Jakobsson J, Malm C, Furberg M, Ekelund U, Svensson M. Physical activity during the coronavirus (COVID-19) pandemic: prevention of a decline in metabolic and immunological functions. Front Sports Active Living. 2020;2:57.

61. Riecher-Rössler A. Sex and gender differences in mental disorders. Lancet Psychiatry. 2017:4(1):8-9.

62. Giustino V, Parroco AM, Gennaro A, Musumeci G, Palma A, Battaglia G. Physical activity levels and related energy expenditure during COVID-19 quarantine among the Sicilian active population: a cross-sectional online survey study. Sustainability. 2020;12(11):4356.

63. Committee IR. Guidelines for data processing and analysis of the International Physical Activity Questionnaire (IPAQ)-short and long forms. http:// www.ipaq.ki.se/scoring.pdf. 2005.

64. Thompson PD, Arena R, Riebe D, Pescatello LS. ACSM's new preparticipation health screening recommendations from ACSM's guidelines for exercise testing and prescription. Curr Sports Med Rep. 2013;12(4):215-7.

65. Neill RD, Lloyd K, Best P, Tully MA. The effects of interventions with physical activity components on adolescent mental health: systematic review and meta-analysis. Ment Health Phys Act. 2020;19:100359.

66. Gan Y, Ma J, Wu J, Chen Y, Zhu H, Hall BJ. Immediate and delayed psychological effects of province-wide lockdown and personal quarantine during the COVID-19 outbreak in China. Psychol Med. 2020:1-12.

67. Sher L. The impact of the COVID-19 pandemic on suicide rates. QJM. 2020;113(10):707-12.

\section{Publisher's Note}

Springer Nature remains neutral with regard to jurisdictional claims in published maps and institutional affiliations.

Ready to submit your research? Choose BMC and benefit from:

- fast, convenient online submission

- thorough peer review by experienced researchers in your field

- rapid publication on acceptance

- support for research data, including large and complex data types

- gold Open Access which fosters wider collaboration and increased citations

- maximum visibility for your research: over $100 \mathrm{M}$ website views per year

At BMC, research is always in progress.

Learn more biomedcentral.com/submissions 